Author affiliations appear at the end of this article.

Published at ascopubs.org/journal/jco on December 5, 2016

Support information appears at the end of this article.

Presented in part at the Multinational Association of Supportive Care in Cancer/ International Society of Oral Oncology Annual Meeting on Supportive Care in Cancer, Copenhagen, Denmark, June 2527, 2015, and International PsychoOncology Society Congress, Dublin, Ireland, October 19-21, 2016.

Clinical trial information: NTR1868.

Corresponding author: Irma M. Verdonck-de Leeuw, PhD, VU Medical Center, PO Box 7057, 1007 MB, Amsterdam,

Netherlands; e-mail: im.verdonck@vumc.nl

C 2016 by American Society of Clinical Oncology

0732-183X/17/3503w-314w/\$20.00

\title{
Cost-Utility of Stepped Care Targeting Psychological Distress in Patients With Head and Neck or Lung Cancer
}

Femke Jansen, Anna M.H. Krebber, Veerle M.H. Coupé, Pim Cuijpers, Remco de Bree, Annemarie Becker-Commissaris, Egbert F. Smit, Annemieke van Straten, Guus M. Eeckhout, Aartjan T.F. Beekman, C. René Leemans, and Irma M. Verdonck-de Leeuw

\section{$\begin{array}{llllllll}\text { A } & \text { B } & \text { S } & \text { T } & \text { R } & \text { A } & \text { C } & \text { T }\end{array}$}

\section{Purpose}

A stepped care (SC) program in which an effective yet least resource-intensive treatment is delivered to patients first and followed, when necessary, by more resource-intensive treatments was found to be effective in improving distress levels of patients with head and neck cancer or lung cancer. Information on the value of this program for its cost is now called for. Therefore, this study aimed to assess the cost-utility of the SC program compared with care-as-usual (CAU) in patients with head and neck cancer or lung cancer who have psychological distress.

\section{Patients and Methods}

In total, 156 patients were randomly assigned to SC or CAU. Intervention costs, direct medical costs, direct nonmedical costs, productivity losses, and health-related quality-of-life data during the intervention or control period and 12 months of follow-up were calculated by using Trimbos and Institute of Medical Technology Assessment Cost Questionnaire for Psychiatry, Productivity and Disease Questionnaire, and EuroQol-5 Dimension measures and data from the hospital information system. The SC program's value for the cost was investigated by comparing mean cumulative costs and quality-adjusted life years (QALYs).

\section{Results}

After imputation of missing data, mean cumulative costs were -€3,950 (95\% Cl, $-€ 8,158$ to $-€ 190)$ lower, and mean number of QALYs was $0.116(95 \% \mathrm{Cl}, 0.005$ to 0.227$)$ higher in the intervention group compared with the control group. The intervention group had a probability of $96 \%$ that cumulative QALYs were higher and cumulative costs were lower than in the control group. Four additional analyses were conducted to assess the robustness of this finding, and they found that the intervention group had a probability of $84 \%$ to $98 \%$ that cumulative QALYs were higher and a probability of $91 \%$ to $99 \%$ that costs were lower than in the control group.

\section{Conclusion}

$\mathrm{SC}$ is highly likely to be cost-effective; the number of QALYs was higher and cumulative costs were lower for SC compared with CAU.

\section{J Clin Oncol 35:314-324. (C) 2016 by American Society of Clinical Oncology}

\section{INTRODUCTION}

Recent reviews on the cost-effectiveness and cost-utility of psychosocial care in patients with cancer in general found that psychosocial care is likely to be cost-effective at potentially acceptable willingness-to-pay thresholds. ${ }^{1,2}$ However, more research is warranted because economic evaluations are scarce, and heterogeneity among studies hampers comparison of the findings. In addition, there have not been any studies specifically targeted to novel psychosocial care programs such as stepped care (SC). To overcome barriers to the use of psychosocial care for patients with cancer, an SC program targeting psychological distress in those patients has been developed, and it consists of four steps: (1) watchful waiting for 2 weeks, (2) guided self-help, (3) face-to-face problem-solving therapy, and (4) specialized psychological interventions (eg, cognitive behavioral therapy) and/or psychotropic medication. ${ }^{3}$ Patients proceed to the next step only when symptoms of distress do not resolve.

Recently, this SC program was found to have beneficial effects on distress compared with 
care-as-usual (CAU) in patients with head and neck cancer (HNC) or lung cancer (LC). ${ }^{4}$ Patients with HNC or LC were targeted because they are seldom involved in randomized controlled trials of psychosocial care, despite a high prevalence of depression. ${ }^{5}$ Previous economic evaluation studies of SC programs targeting primary care patients, ${ }^{6-9}$ older patients, ${ }^{10,11}$ patients with diabetes, ${ }^{12,13}$ or patients with acute coronary syndrome ${ }^{14}$ who have psychological distress have found that, except in one study, ${ }^{10}$ the SC program improved quality-adjusted life years (QALYs) or depression-free days compared with control care, albeit at higher cost in most studies. ${ }^{6,7,9,11,13}$ However, no such economic evaluation of SC has yet been performed in patients with cancer. This study therefore assessed the cost-utility of an SC program targeting psychological distress in patients with HNC or LC compared with patients who received CAU.

\section{PATIENTS AND METHODS}

\section{Study Design and Population}

Detailed information on the study design and population can be found in previous publications. ${ }^{3,4}$ In short, this cost-utility analysis was conducted alongside a prospective randomized controlled trial on the efficacy of an SC program for patients with HNC or LC who had symptoms of psychological distress, a Hospital Anxiety and Depression Scale (HADS) distress score $>14$, or an anxiety or depression score $>7$. The study was approved by the Medical Ethics Committee of VU Medical Center and conducted according to the principles of the Declaration of Helsinki.

\section{Randomization and Treatment Allocation}

Patients who met the eligibility criteria and signed informed consent were randomly assigned to the intervention group that was given $\mathrm{SC}$ or to the control group that was given CAU (Fig 1). The SC program consisted of four steps: (1) watchful waiting, (2) guided self-help via the Internet or a booklet, (3) face-to-face problem-solving therapy, and (4) specialized psychological interventions and/or psychotropic medication. Patients who did not recover after a treatment step (HADS for anxiety/depression score remained above 7) proceeded to a more intensive step. More information is provided in the Data Supplement. ${ }^{3}$

\section{Outcome Measures}

Cost and clinical end point data were collected at baseline (t0), immediately after the intervention or control period (t1), and at 3, 6, 9, and 12 months after $t 1$. The economic evaluation was conducted from a societal perspective and included intervention costs, direct medical costs (cost of health care use and medication), direct nonmedical costs (cost of support groups, informal care, traveling to health services, and parking), and indirect nonmedical costs (loss of productivity from paid work). Intervention costs were calculated by using a bottom-up approach. Mean costs per patient in the intervention group were $€ 318$ (range, $€ 24$ to $€ 9,043$; Table 1).

The Trimbos and Institute of Medical Technology Assessment Cost Questionnaire for Psychiatry (TiC-P) ${ }^{15}$ was used to measure the use of health care facilities (eg, number of visits to the general practitioner) and other facilities (eg, time spent in self-help groups or informal care) in the past 4 weeks and medication used (antidepressants, analgesics, and sedatives) in the past 2 weeks. In addition, data on health care use within the hospital (visits to the medical specialist, day treatment, and hospital admission) was collected by using the hospital information system. Direct medical and direct nonmedical costs of support groups and informal care were calculated by multiplying resource use by the integral cost price. ${ }^{16}$ Direct nonmedical costs of traveling to health services and parking were calculated by multiplying unit resource use by average distance to the location times the price per kilometer. All prices were adjusted to 2011 prices by using the consumer price index.

The Productivity and Disease Questionnaire (PRODISQ) ${ }^{17}$ was used to measure productivity losses through absence from paid work (absenteeism) or reduced quantity or quality of performed paid work (presenteeism) in the past 4 weeks. Losses as a result of presenteeism were calculated by multiplying the days of less productivity at work by the estimated amount of lost quantity or quality of performed work (ranging from 0 to 10 points). Indirect nonmedical costs from paid work were calculated by multiplying productivity losses by age- and sex-specific $\operatorname{costs}^{16}$ using the human capital approach. The EuroQol-5 Dimension (EQ-5D) instrument was used to measure health-related quality of life. The EQ-5D utility score was obtained by using the Dutch index tariff. ${ }^{18}$

\section{Statistical Analyses}

All analyses were performed by using SPSS version 20 (IBM, Armonk, NY) and STATA version 12.1 (STATA, College Station, TX). Descriptive statistics, $\chi^{2}$ tests, and independent $t$ tests were used to describe and compare baseline characteristics among different groups.

To provide information on types of costs included in the analyses and their relative importance (their contribution to the mean total costs per group) at various time points, data for complete cases (patients who completed the baseline measurement and all five follow-up measurements or who completed the baseline measurement and all follow-up measurements until they died) were used. Data for complete cases were also used to provide information on the mean utility scores per group at the different time points.

To assess the value of SC for its cost compared with CAU, a base case intention-to-treat cost-utility analysis was performed at first that included all 156 randomly assigned patients and imputed any missing data. Consequently, to assess the robustness of this finding, four additional analyses were performed: (1) an analysis in which we adjusted the base case analysis by using multivariable regression analyses for variables at baseline found to have a major influence (a change of $\geq 20 \%$ ) on incremental costs (European Organisation for Research and Treatment of Cancer [EORTC] social functioning and total costs at baseline) and incremental effects (HADS for depression at baseline), (2) an analysis excluding patients from the base case analysis who died during the study, (3) an analysis in which data were imputed for patients who died during the study as though they were still alive, and (4) an analysis in which productivity losses were excluded.

All cost-utility analyses were performed in agreement with the intention-to-treat principle. Missing data were imputed as total costs or utility score per time point per treatment arm separately by using multiple imputation (predictive mean matching) by chained equations. Data were thus imputed only for those time points that were missing. Linear and logistic regression analyses were performed to investigate which variables (sociodemographic, clinical, HADS total, HADS for depression, HADS for anxiety, and EORTC global quality of life) were associated with missing data, observed costs, or EQ-5D utility scores. Variables associated with missing data (sex and HADS total), observed costs (work situation, EORTC global quality of life, and marital status), or utility scores (HADS total, EORTC global quality of life score, tumor stage, tumor location, and years of education) and variables that differed at baseline (alcohol dependency, HADS-D, EORTC Quality of Life Questionnaire-Core 30 [EORTC QLQC30] social functioning, and EORTC module for head and neck cancer [EORTC QLQ-H\&N35] social contact and sexuality) were included in the multiple imputation model. Ten imputed data sets were created and analyzed separately. Results of the 10 analyses were pooled by using Rubin's (1987) rules.

To perform incremental cost-utility analyses, the cumulative costs and number of QALYs per patient per treatment group were calculated. For patients in the control group, cumulative costs as measured by using the TiC-P and PRODISQ between $\mathrm{t} 0$ and $\mathrm{t} 1$ were calculated by multiplying the mean costs at time point $\mathrm{tl}$ by the corresponding time period (time between $\mathrm{t} 0$ and $\mathrm{t} 1$ ). Unlike patients in the control group, for patients randomly assigned to the intervention group, the costs as measured by 


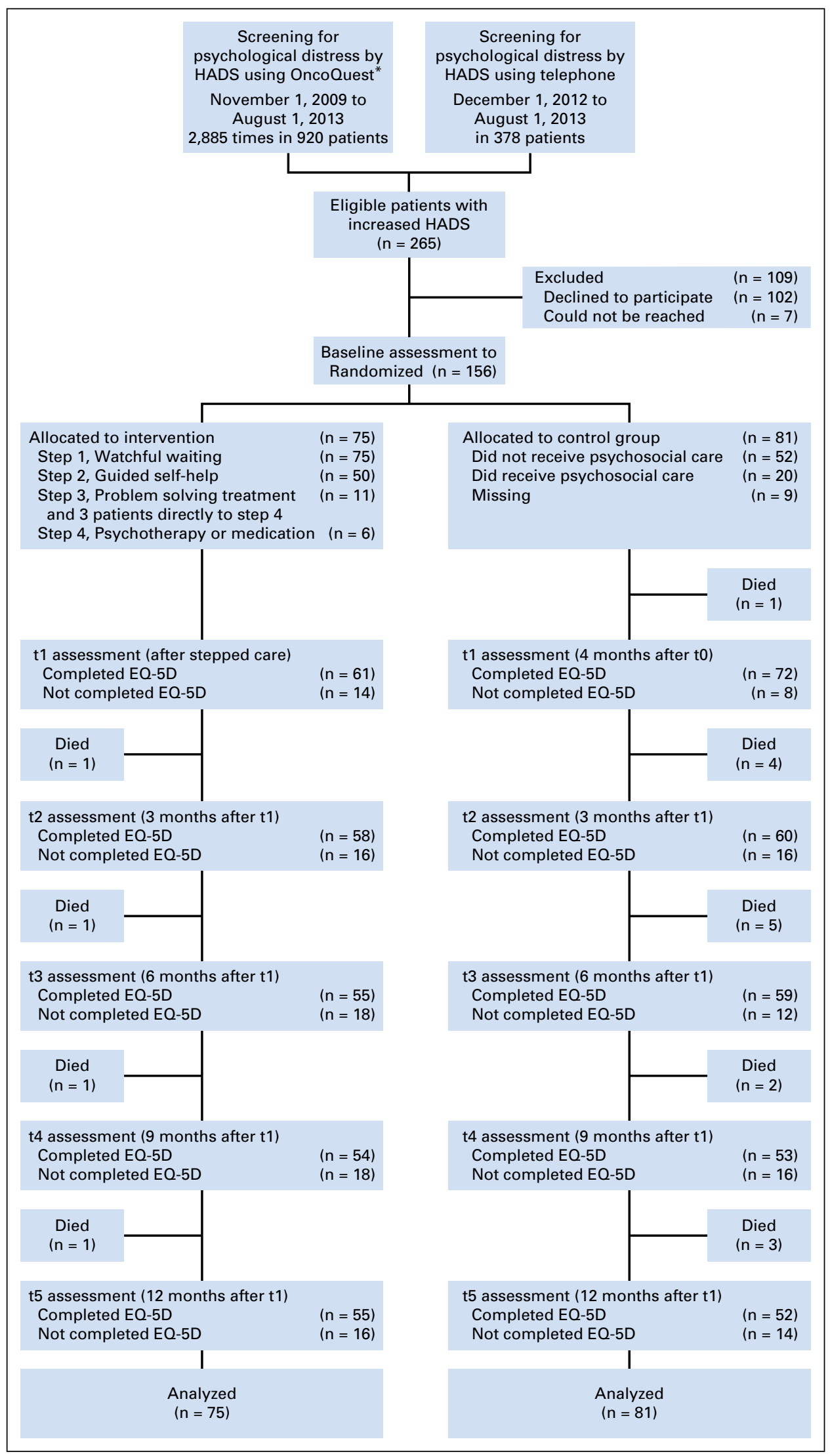




\begin{tabular}{|c|c|}
\hline Steps & Cost $(€)$ \\
\hline \multicolumn{2}{|l|}{ Screening } \\
\hline Screening for distress & 7.97 \\
\hline Consultation with a nurse for 15 minutes & 7.97 \\
\hline \multicolumn{2}{|l|}{ Step 1: Watchful waiting } \\
\hline Monitoring distress with HADS assessment & 7.97 \\
\hline \multicolumn{2}{|l|}{ Step 2: Guided self-help } \\
\hline Self-help Internet tool or booklet & 39.00 \\
\hline Feedback from a nurse by e-mail or telephone (in 1 hour total) & 31.88 \\
\hline Monitoring distress with HADS assessment & 7.97 \\
\hline \multicolumn{2}{|l|}{ Step 3: Face-to-face problem-solving therapy } \\
\hline Five sessions of problem-solving therapy with a nurse & 151.42 \\
\hline Monitoring distress with HADS assessment & 7.97 \\
\hline \multicolumn{2}{|l|}{$\begin{array}{l}\text { Step 4: Specialized psychological interventions and/or } \\
\text { psychotropic medication }\end{array}$} \\
\hline $\begin{array}{l}\text { Costs were calculated per person individually because type } \\
\text { of treatment and number of sessions or duration of } \\
\text { treatment differed. }\end{array}$ & Differed \\
\hline
\end{tabular}

Abbreviation: HADS, Hospital Anxiety and Depression Scale. using the TiC-P and PRODISQ at $\mathrm{t} 1$ were not expected to be generalizable to the entire intervention period (a patient was expected to have different costs during step 4 than during step 1). Therefore, cumulative costs between $\mathrm{t} 0$ and $\mathrm{t} 1$ for intervention group patients were calculated by summing costs per step. Mean costs per step per 4 weeks were calculated for all patients who participated in step 4 , for patients who participated in step 3 but not in step 4, for patients who participated in step 2 but not in steps 3 or 4 , and for patients who participated in step 1 but not in steps 2, 3, or 4. Subsequently, cumulative costs per patient were calculated by multiplying mean cumulative costs per step per 4 weeks by the time a patient participated in the particular step. Costs between t 1 and t5 as measured by using the TiC-P and PRODISQ for both groups were calculated by using linear interpolation. Total cumulative costs per patient were calculated by summing cumulative costs measured by using the TiC-P and PRODISQ with intervention costs and costs measured by using the hospital information system. The number of QALYs per patient was calculated by multiplying the EQ-5D utility score by the appropriate time period it accounts for by using linear interpolation.

An incremental cost-utility ratio was calculated to obtain the costs per gained QALY by dividing the incremental costs by the incremental effects by using the following formula (mean costs $s_{\text {intervention }}$ - mean $\left.\operatorname{costs}_{\text {control }}\right) /$

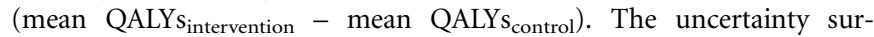
rounding the incremental cost-utility ratio was assessed by using bootstrapping with 5,000 replications and was projected on a cost-utility plane.

\section{RESULTS}

In total, 75 patients were randomly assigned to the intervention group and 81 patients to the control group. Table 2 summarizes the baseline characteristics of both groups and compares patients with complete data with patients who did not have complete data. During the study, four $(5.3 \%)$ of 75 patients in the intervention group versus $15(18.5 \%)$ of 81 patients in the control group died $(P=.012)$.

\section{Direct and Indirect Medical and Productivity Costs}

The mean costs for patients with complete data (patients who completed the baseline measurement and all five follow-up measurements or who completed the baseline measurement and all follow-up measurements until they died) per time point per group are presented in Table 3 . In the 4 weeks before baseline measurement, no statistically significant differences in costs were found between the two groups $(P=.17)$, although there were large absolute differences. Mean total costs at baseline in the intervention group were $€ 660$ (standard deviation, $€ 1,150$ ) compared with $€ 1,087$ (standard deviation, $€ 1,958$ ) in the control group.

\section{Health-Related Quality of Life}

Table 4 presents the mean EQ-5D utility score for patients with complete data. At baseline, a statistically nonsignificant difference in EQ-5D utility score of .08 was found in favor of the intervention group $(P=.12)$, which exceeded the subjectively appreciable difference of .07 reported in Walters et al. ${ }^{19}$

\section{Cost-Utility Analyses}

Results of the different cost-utility analyses are presented in Table 5 and Figure 2. In the base case analysis, mean costs in the intervention group were statistically significantly lower than mean costs in the control group (incremental costs were $-€ 3,950$ ). Besides, QALYs gained were statistically significantly higher in the intervention group compared with the control group (incremental effects were .116). Of the bootstrapped cost-utility pairs, $96 \%$ fell into the southeast quadrant, representing the probability that SC is more effective and less costly compared with CAU.

To assess the robustness of this finding, four additional analyses were performed as presented in Table 5 . In these additional analyses, the intervention group had a probability of $84 \%$ to $98 \%$ that cumulative QALYs were higher and a probability of $91 \%$ to $99 \%$ to be less costly than the control group. The analysis that showed the lowest probability of being more effective and less costly was the analysis in which patients who died during the study were excluded (probability of $81 \%$ ).

\section{DISCUSSION}

This study investigated the cost-utility of an SC program targeting psychological distress in patients with HNC or LC compared with CAU. In the base case analysis, the number of QALYs was statistically significantly higher and cumulative costs were statistically significantly lower in the intervention group compared with the control group. The probability that cumulative QALYs were higher and costs were lower was $96 \%$, indicating that SC is highly likely to be cost-effective compared with CAU.

Several additional analyses were performed to assess the robustness of this finding. In one analysis, we adjusted for variables that differed at baseline between the two groups and that had a major impact on incremental costs or incremental effects. After correction, incremental costs and QALYs decreased to a statistically nonsignificant difference; however, the intervention group still had a probability of $93 \%$ that cumulative QALYs were higher and a probability of $89 \%$ that they were less costly than the control group.

In addition, we investigated the influence of the lower mortality rate in the intervention compared with the control group $(5.3 \% \vee 18.5 \%)$. A debate is ongoing concerning the influence of psychosocial care on survival in patients with cancer; some authors suggest that psychosocial care may improve survival, ${ }^{20-22}$ although 


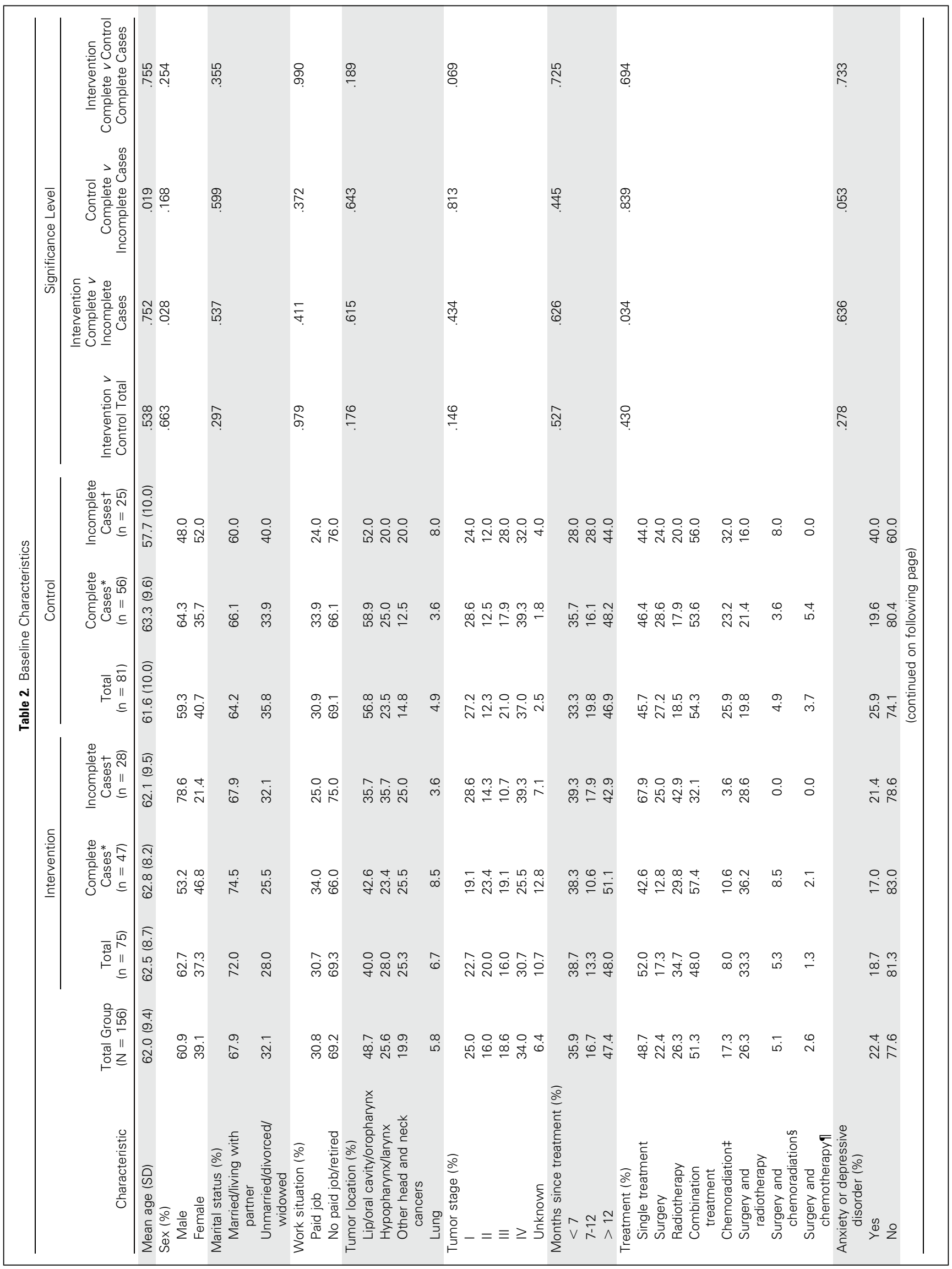




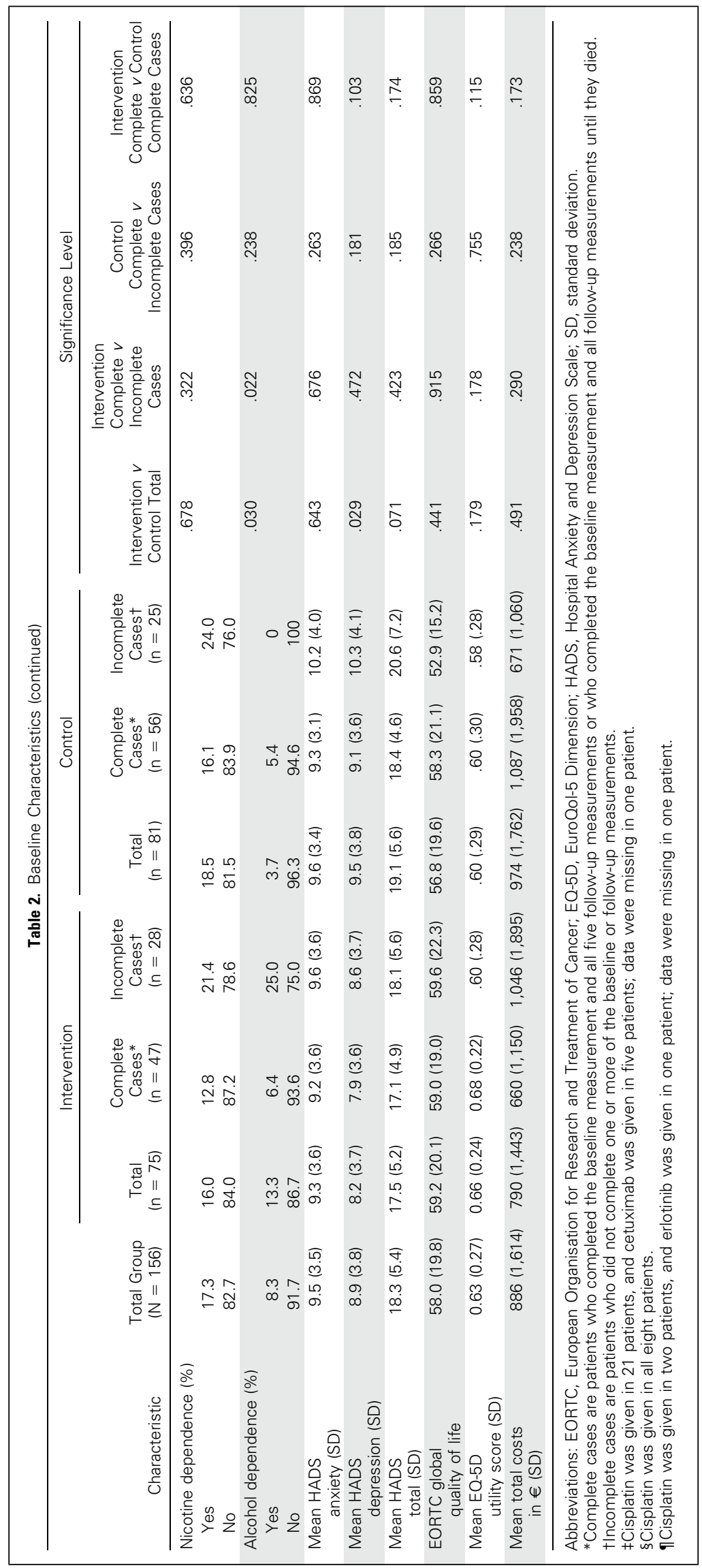




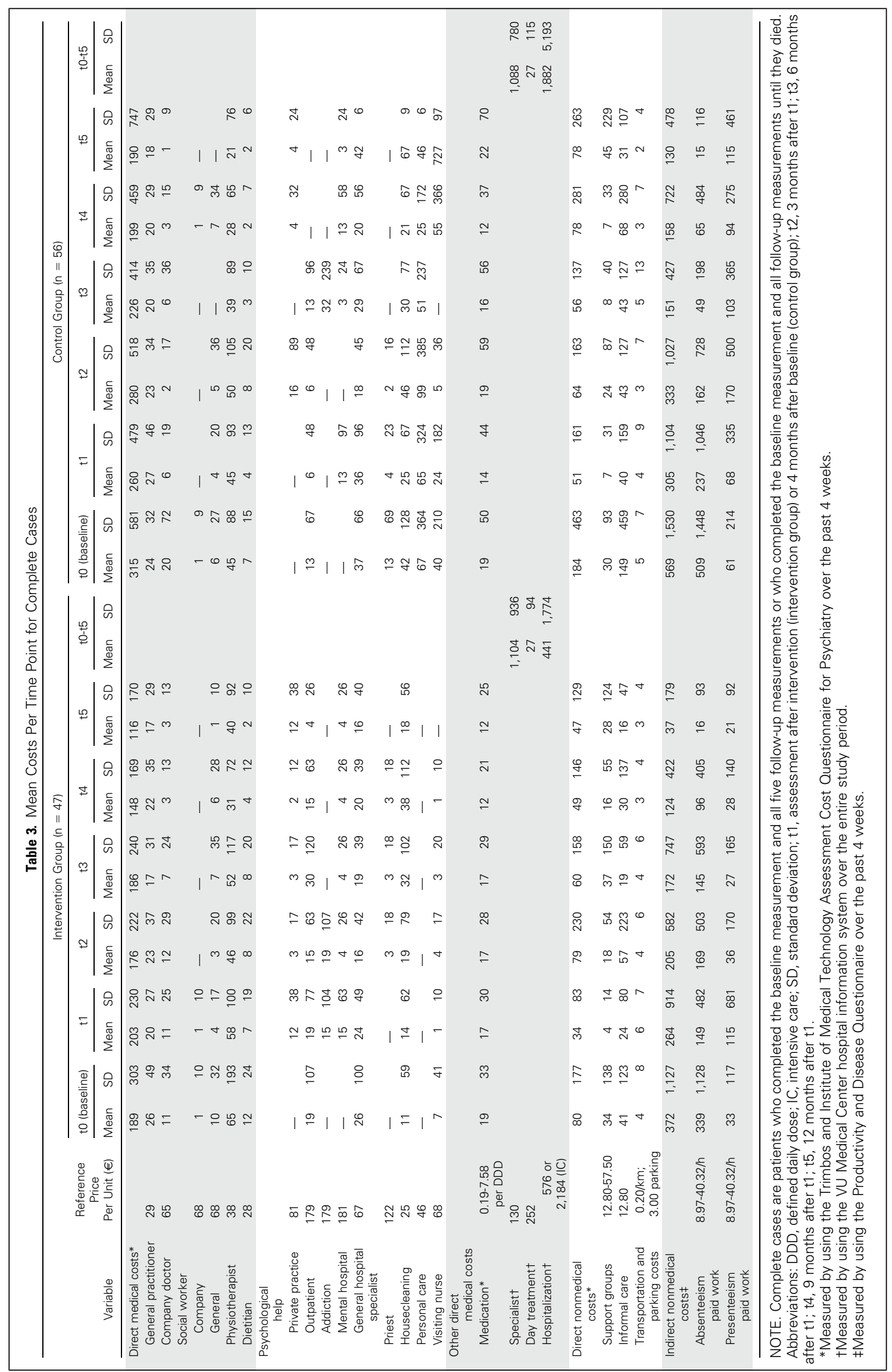


Table 4 Mean EQ-5D Utility Score Per Time Point for Complete Cases

\begin{tabular}{|c|c|c|c|c|}
\hline \multirow[b]{2}{*}{ Time Point } & \multicolumn{2}{|c|}{$\begin{array}{c}\text { Intervention } \\
\text { Group } \\
(\mathrm{n}=47)\end{array}$} & \multicolumn{2}{|c|}{$\begin{array}{l}\text { Control Group } \\
\quad(n=56)\end{array}$} \\
\hline & Mean & SD & Mean & SD \\
\hline Baseline T1 & .68 & .22 & .60 & .30 \\
\hline t1 & .74 & .20 & .65 & .30 \\
\hline t2 (3 months after t1) & .77 & .19 & .65 & .30 \\
\hline t3 (6 months after t1) & .75 & .20 & .61 & .32 \\
\hline t4 (9 months after t1) & .74 & .24 & .61 & .35 \\
\hline t5 (12 months after t1) & .73 & .22 & .60 & .36 \\
\hline
\end{tabular}

NOTE. Complete cases are patients who completed the baseline measurement and all five follow-up measurements or who completed the baseline measurement and all follow-up measurements until they died. t1, assessment after the intervention (intervention group) or 4 months after baseline (control group). Abbreviation: EQ-5D, EuroQol-5 Dimension.

others argue against such an effect. ${ }^{23-25}$ If we assume that psychosocial care does not improve survival, our cost-utility estimate may be biased, because the higher mortality rate in the control group would have resulted in lower mean QALYs and was expected to influence mean total costs. Therefore, two additional analyses were performed: one analysis in which all patients who died were excluded and one analysis in which for all patients who died, cost and utility data were imputed as though they were still alive. In both analyses, incremental costs between the two groups changed somewhat, while the incremental QALYs decreased to a statistically nonsignificant difference. However, the intervention group still had a probability of $84 \%$ to $90 \%$ that cumulative QALYs were higher than in the control group and a probability of $96 \%$ to $99 \%$ that the intervention was less costly. This indicates that when SC does not influence survival, it is still likely to be cost-effective.

Our findings are in agreement with those from one previous study that targeted patients with cancer who had increased levels of distress. ${ }^{26}$ All other previous studies targeting patients with cancer who had increased levels of distress reported an improvement in QALYs, although at higher costs. ${ }^{27-29}$ This difference in cost-benefit may be a result of the design of SC in which intervention patients are first provided with watchful waiting (recovery rate, 28\%), followed by guided self-help when they do not spontaneously recover after 2 weeks (recovery rate, $34 \%) .{ }^{4}$ When the patient had still not recovered after guided self-help, more resource-intensive care was provided, although in the previous studies, all patients in the intervention group received relatively more resource-intensive care. ${ }^{26-29}$

Another explanation for the difference in cost-benefit may be that, unlike previous studies, ${ }^{26-29}$ our study was conducted from a societal perspective, and it incorporated productivity losses and direct nonmedical costs such as informal care costs. Previous studies found that being distressed was associated with unemployment in survivors of mixed cancer types ${ }^{30}$ and that higher levels of depression were associated with unemployment as a result of loss of job, sick leave, or early retirement after treatment for HNC. ${ }^{31}$ In another study of employment and return to work among patients with HNC, an association between anxiety and return to work was reported, although no association with distress or depression was found. ${ }^{32}$ Our efficacy study showed that SC was beneficial in improving level of distress, ${ }^{4}$ which may have had a beneficial effect on productivity losses in the intervention group compared with the control group. We conducted an additional analysis in which we excluded productivity losses; indeed, the analysis showed that the cost difference between the two groups was reduced by $€ 1,062$. However, even without productivity losses, SC had a probability of $97 \%$ to be more effective and less costly.

Some potential limitations were evident in this study. One potential limitation is that several assumptions were made regarding resource use and EQ-5D utility scores for data that were missing. First, missing total costs or utility scores per time point per treatment were imputed using multiple imputation techniques. Second, linear interpolation between time points was used. Both assumptions may not necessarily reflect reality, however, because the same assumptions were made for both groups, this was not expected to have influenced our findings. Another potential limitation is that productivity losses were calculated using the human capital approach instead of the recommended friction cost approach. ${ }^{16}$ The small sample size of 156 patients is another limitation of this study. Although bootstrapping was

\begin{tabular}{|c|c|c|c|c|c|c|c|c|c|}
\hline \multirow[b]{2}{*}{ Analysis and Group } & \multirow[b]{2}{*}{ No. of Patients } & \multicolumn{2}{|c|}{ Costs $(€)$} & \multicolumn{2}{|c|}{ QALY } & \multicolumn{2}{|c|}{ Incremental Costs } & \multicolumn{2}{|c|}{ Incremental Effects } \\
\hline & & Mean & SEM & Mean & SEM & $€$ & $95 \% \mathrm{Cl}$ & QALY & $95 \% \mathrm{Cl}$ \\
\hline Base case & & & & & & $-3,950$ & $-8,158$ to $-190^{*}$ & .116 & .005 to $.227^{*}$ \\
\hline Intervention & 75 & 9,761 & 1,041 & .884 & .039 & & & & \\
\hline Control & 81 & 13,711 & 1,828 & .768 & .040 & & & & \\
\hline Adjusted for several variables at baseline & & & & & & $-2,499$ & $-6,082$ to 630 & .076 & -.032 to .184 \\
\hline Intervention & 75 & NA & & NA & & & & & \\
\hline Control & 81 & NA & & NA & & & & & \\
\hline Excluding patients who died during the study & & & & & & $-3,939$ & $-8,722$ to 229 & .052 & -.053 to .156 \\
\hline Intervention & 71 & 9,934 & 1,088 & .911 & .037 & & & & \\
\hline Control & 66 & 13,874 & 2,080 & .859 & .038 & & & & \\
\hline With imputed data for patients who died during the study & & & & & & $-4,692$ & $-8,898$ to $-889 *$ & .059 & -.035 to .153 \\
\hline Intervention & 75 & 9,887 & 1,035 & .908 & .035 & & & & \\
\hline Control & 81 & 14,579 & 1,848 & .849 & .033 & & & & \\
\hline Without productivity losses & & & & & & $-2,888$ & $-5,630$ to $-424^{*}$ & .118 & .009 to $.227^{*}$ \\
\hline Intervention & 75 & 6,287 & 677 & .885 & .039 & & & & \\
\hline Control & 81 & 9,175 & 1,161 & .767 & .040 & & & & \\
\hline
\end{tabular}

Abbreviations: NA, not applicable; QALY, quality-adjusted life year.

* Significant difference between the two groups $(P<.05)$. 


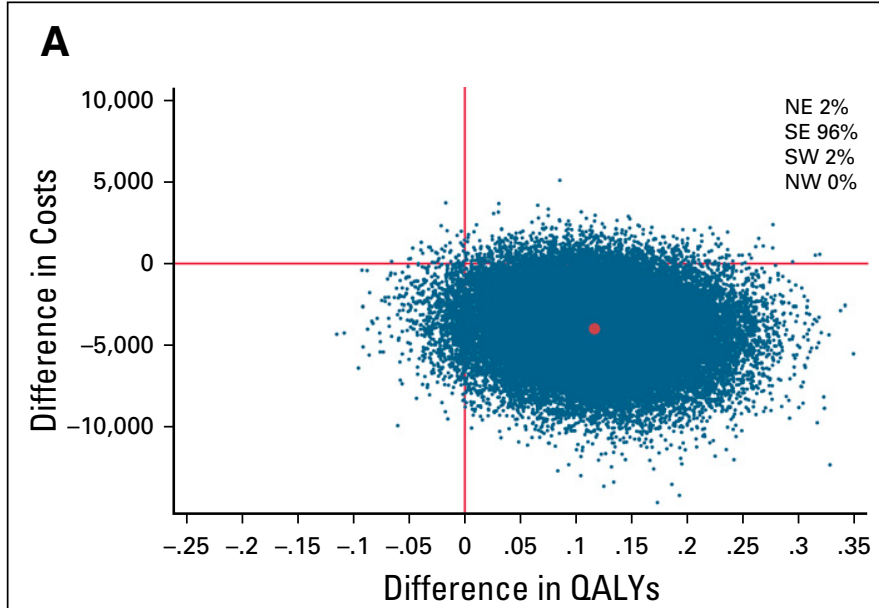

\section{B}

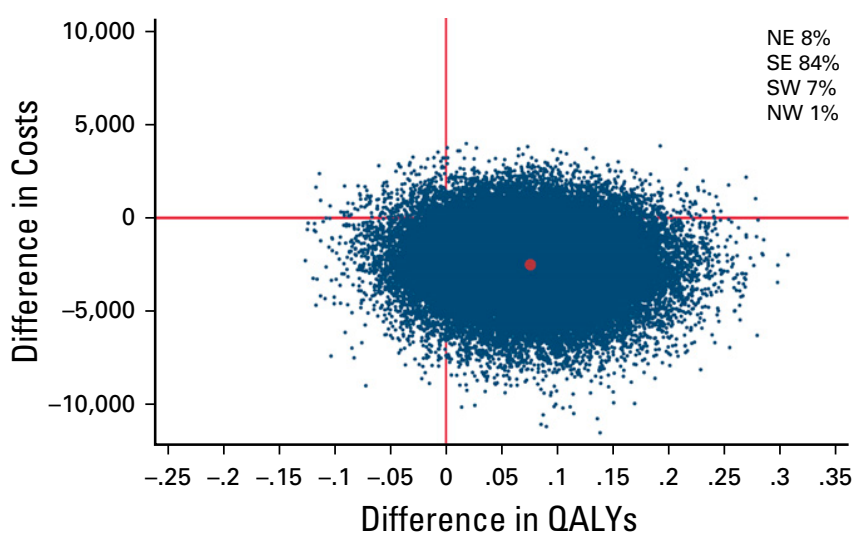

C
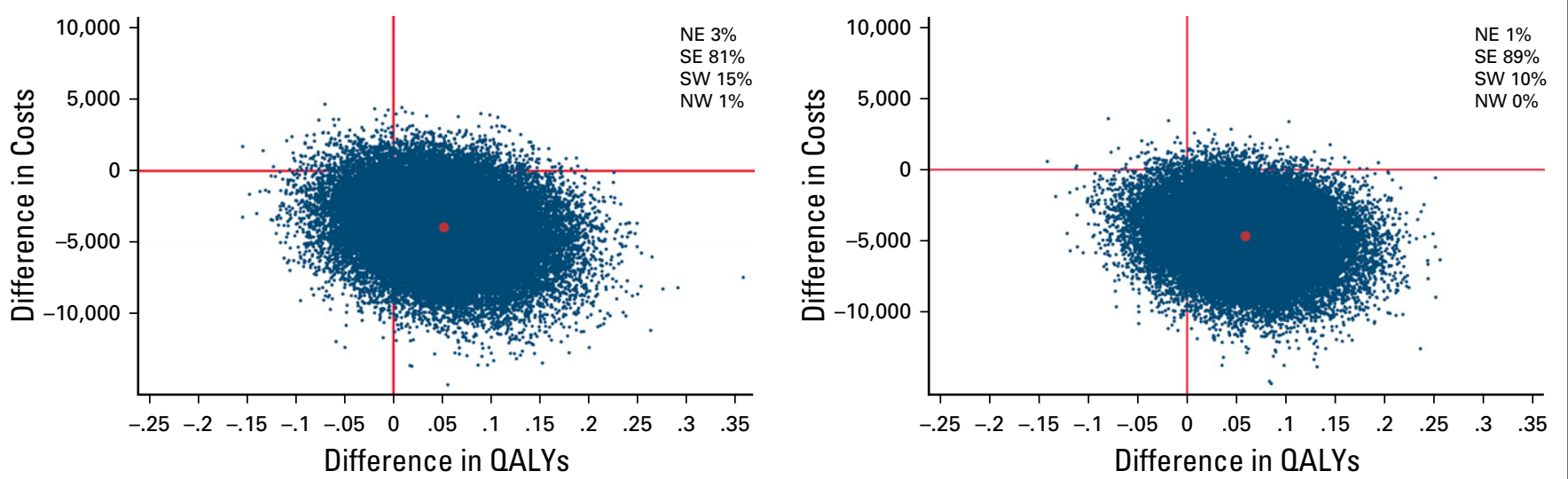

$\mathbf{E}$

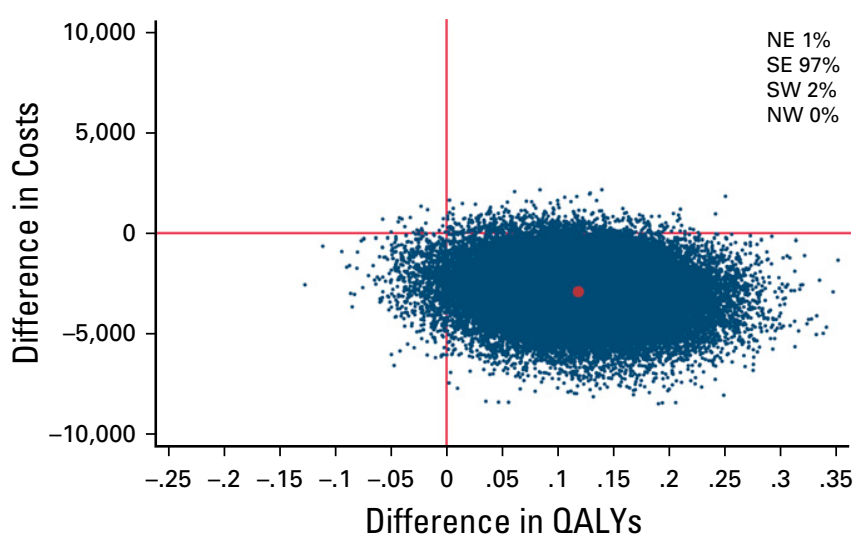

Fig 2 Cost-utility plane of (A) the base case analysis (B) adjusted for social functioning and total costs at baseline (costs) and Hospital Anxiety and Depression Scale (effects), (C) without patients who died during the study, (D) with imputed data for patients who died during the study, and (E) with productivity losses not included. QALYs, quality-adjusted life years. The percentages indicate the percentage of bootstrap replications in a certain quadrant.

performed, which supported the finding that SC is likely to be more effective and less costly than CAU, it also showed that there is considerable uncertainty. More research is therefore needed on the costutility of SC in subgroups of the investigated population, such as in patients with and without a diagnosis of major depression disorder or anxiety disorder.
Further research should investigate whether findings are replicable in other groups of patients with cancer. Further research should also be performed on optimal implementation of SC in routine cancer care, which may potentially differ between different health care systems (eg, the Netherlands compared with the United States). The Reach, Efficacy, Adoption, Implementation, and 
Maintenance framework can be used to evaluate the different steps involved in optimal implementation and maintenance of SC. ${ }^{33}$

In the base case analysis, the number of QALYs was statistically significantly higher and cumulative costs were statistically significantly lower in the intervention group compared with the control group; thus, SC is highly likely to be dominant (more effective and less costly) compared with CAU. After adjusting for differences at baseline, taking into account differences in mortality rate and excluding productivity losses, the number of QALYs and cumulative costs mostly decreased to a statistically nonsignificant difference. However, the intervention group still had a probability of $84 \%$ to $98 \%$ that cumulative QALYs were higher and a probability of $91 \%$ to $99 \%$ that they would be less costly than those in the control group, supporting the finding that SC is likely to be costeffective. In combination with findings on the efficacy of SC, ${ }^{4} \mathrm{SC}$ is expected to be beneficial in routine HNC and LC care practice. Further research is needed on the optimal implementation of this SC program in clinical practice.

\section{AUTHORS' DISCLOSURES OF POTENTIAL CONFLICTS} OF INTEREST

Disclosures provided by the authors are available with this article at ascopubs.org/journal/jco.

\section{AUTHOR CONTRIBUTIONS}

Conception and design: Pim Cuijpers, Remco de Bree, Annemarie BeckerCommissaris, Egbert F. Smit, Annemieke van Straten, Guus M. Eeckhout, Aartjan T.F. Beekman, C. René Leemans, Irma M. Verdonck-de Leeuw Collection and assembly of data: Femke Jansen, Anna M.H. Krebber, Remco de Bree, Annemarie Becker-Commissaris, Egbert F. Smit, C. René Leemans, Irma M. Verdonck-de Leeuw

Data analysis and interpretation: Femke Jansen, Veerle M.H. Coupé, C. René Leemans, Irma M. Verdonck-de Leeuw

Manuscript writing: All authors

Final approval of manuscript: All authors

Accountable for all aspects of the work: All authors

\section{REFERENCES}

1. Jansen F, van Zwieten V, Coupé VM, et al: A review on cost-effectiveness and cost-utility of psychosocial care in cancer patients. Asia-Pacific $J$ Oncol Nurs 3:125-136, 2016

2. Dieng $M$, Cust $A E$, Kasparian NA, et al: Economic evaluations of psychosocial interventions in cancer: $A$ systematic review. Psychooncology, 2016 doi:10.1002/ pon.4075 [epub ahead of print on January 26, 2016]

3. Krebber AM, Leemans CR, de Bree $R$, et al: Stepped care targeting psychological distress in head and neck and lung cancer patients: A randomized clinical trial. BMC Cancer 12:173, 2012

4. Krebber AM, Jansen F, Witte BI, et al: Stepped care targeting psychological distress in head and neck cancer and lung cancer patients: A randomized, controlled trial. Ann Oncol 27:1754-1760, 2016

5. Krebber AM, Buffart LM, Kleijn G, et al: Prevalence of depression in cancer patients: $A$ meta-analysis of diagnostic interviews and selfreport instruments. Psychooncology 23:121-130, 2014

6. Katon WJ, Schoenbaum M, Fan MY, et al: Costeffectiveness of improving primary care treatment of latelife depression. Arch Gen Psychiatry 62:1313-1320, 2005

7. Araya R, Flynn T, Rojas G, et al: Costeffectiveness of a primary care treatment program for depression in low-income women in Santiago, Chile. Am J Psychiatry 163:1379-1387, 2006

8. Buttorff $C$, Hock RS, Weiss HA, et al: Economic evaluation of a task-shifting intervention for common mental disorders in India. Bull World Health Organ 90:813-821, 2012

9. Goorden M, Muntingh $A$, van Marwijk $H$, et al: Cost utility analysis of a collaborative stepped care intervention for panic and generalized anxiety disorders in primary care. J Psychosom Res 77:57-63, 2014

10. Bosmans JE, Dozeman E, van Marwijk HW, et al: Cost-effectiveness of a stepped care programme to prevent depression and anxiety in residents in homes for the older people: A randomised controlled trial. Int J Geriatr Psychiatry 29:182-190, 2014

11. Van't Veer-Tazelaar $P$, Smit $F$, van Hout $H$, et al: Cost-effectiveness of a stepped care intervention to prevent depression and anxiety in late life: Randomised trial. Br J Psychiatry 196:319-325, 2010

12. Simon GE, Katon WJ, Lin EH, et al: Costeffectiveness of systematic depression treatment among people with diabetes mellitus. Arch Gen Psychiatry 64:65-72, 2007

13. Hay JW, Katon WJ, Ell K, et al: Costeffectiveness analysis of collaborative care management of major depression among low-income, predominantly Hispanics with diabetes. Value Health 15:249-254, 2012

14. Ladapo JA, Shaffer JA, Fang $Y$, et al: Costeffectiveness of enhanced depression care after acute coronary syndrome: Results from the Coronary Psychosocial Evaluation Studies randomized controlled trial. Arch Intern Med 172:1682-1684, 2012

15. Bouwmans $C$, De Jong $K$, Timman $R$, et al: Feasibility, reliability and validity of a questionnaire on healthcare consumption and productivity loss in patients with a psychiatric disorder (TiC-P). BMC Health Serv Res 13:217, 2013

16. Hakkaart-van Roijen L, Tan SS, Bouwmans CA: Guideline for cost research: Methods and standard cost prices for economic evaluations in healthcare [in Dutch]. Diemen, the Netherlands: The College for Health Care Insurances, 2010

17. Koopmanschap MA: PRODISQ: A modular questionnaire on productivity and disease for economic evaluation studies. Expert Rev Pharmacoecon Outcomes Res 5:23-28, 2005

18. Lamers LM, Stalmeier PF, McDonnell J, et al: [Measuring the quality of life in economic evaluations: the Dutch EQ-5D tariff] [in Dutch]. Ned Tijdschr Geneeskd 149:1574-1578, 2005

19. Walters SJ, Brazier JE: Comparison of the minimally important difference for two health state utility measures: EQ-5D and SF-6D. Qual Life Res 14: 1523-1532, 2005

20. Spiegel $\mathrm{D}$ : Minding the body: Psychotherapy and cancer survival. Br J Health Psychol 19:465-485, 2014

21. Spiegel D: Effects of psychotherapy on cancer survival. Nat Rev Cancer 2:383-389, 2002

22. Xia $Y$, Tong G, Feng $R$, et al: Psychosocial and behavioral interventions and cancer patient survival again: Hints of an adjusted meta-analysis. Integr Cancer Ther 13:301-309, 2014
23. Boesen EH, Johansen C: Impact of psychotherapy on cancer survival: Time to move on? Curr Opin Oncol 20:372-377, 2008

24. Chow E, Tsao MN, Harth T: Does psychosocial intervention improve survival in cancer? A metaanalysis. Palliat Med 18:25-31, 2004

25. Kissane D: Beyond the psychotherapy and survival debate: The challenge of social disparity, depression and treatment adherence in psychosocial cancer care. Psychooncology 18: 1-5, 2009

26. Chatterton ML, Chambers $S$, Occhipinti $S$, et al: Economic evaluation of a psychological intervention for high distress cancer patients and carers: Costs and quality-adjusted life years. Psychooncology 25:857-864, 2016

27. Strong $V$, Waters $R$, Hibberd $C$, et al: Management of depression for people with cancer (SMaRT oncology 1): A randomised trial. Lancet 372: 40-48, 2008

28. Choi Yoo SJ, Nyman JA, Cheville $A L$, et al: Cost effectiveness of telecare management for pain and depression in patients with cancer: Results from a randomized trial. Gen Hosp Psychiatry 36:599-606, 2014

29. Duarte A, Walker J, Walker S, et al: Costeffectiveness of integrated collaborative care for comorbid major depression in patients with cancer. J Psychosom Res 79:465-470, 2015

30. Tevaarwerk AJ, Lee JW, Sesto $M E$, et al: Employment outcomes among survivors of common cancers: The Symptom Outcomes and Practice Patterns (SOAPP) study. J Cancer Surviv 7:191-202, 2013

31. Koch R, Wittekindt $C$, Altendorf-Hofmann A, et al: Employment pathways and work-related issues in head and neck cancer survivors. Head Neck 37: 585-593, 2015

32. Verdonck-de Leeuw IM, van Bleek WJ, Leemans CR, et al: Employment and return to work in head and neck cancer survivors. Oral Oncol 46:56-60, 2010

33. Glasgow RE, Vogt TM, Boles SM: Evaluating the public health impact of health promotion interventions: The RE-AIM framework. Am J Public Health 89:1322-1327, 1999 


\section{Affiliations}

Femke Jansen, Anna M.H. Krebber, Veerle M.H. Coupé, Annemarie Becker-Commissaris, Egbert F. Smit, Guus M. Eeckhout, Aartjan T.F. Beekman, C. René Leemans, and Irma M. Verdonck-de Leeuw, VU Medical Center; Pim Cuijpers, Annemieke van Straten, and Irma M. Verdonck-de Leeuw, Vrije Universiteit Amsterdam, Amsterdam; and Remco de Bree, University Medical Center Utrecht Cancer Center, Utrecht, Netherlands.

\section{Support}

Supported by Grant No. 300020012 from the Netherlands Organization for Health Research and Development.

\section{Follow ASCO Journals on Twitter}

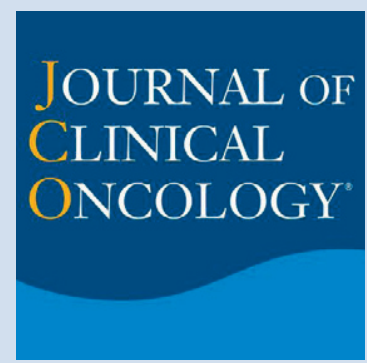

@JCO_ASCO

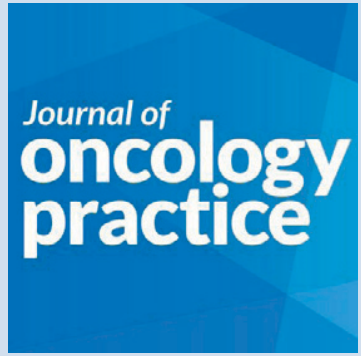

@JOP_ASCO

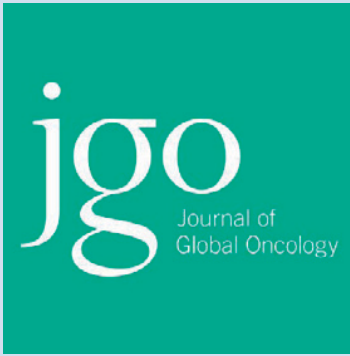

@JGO_ASCO 


\section{AUTHORS' DISCLOSURES OF POTENTIAL CONFLICTS OF INTEREST}

Cost-Utility of Stepped Care Targeting Psychological Distress in Patients With Head and Neck or Lung Cancer

The following represents disclosure information provided by authors of this manuscript. All relationships are considered compensated. Relationships are self-held unless noted. I = Immediate Family Member, Inst = My Institution. Relationships may not relate to the subject matter of this manuscript. For more information about ASCO's conflict of interest policy, please refer to www.asco.org/rwc or ascopubs.org/jco/site/ifc.

\section{Femke Jansen}

No relationship to disclose

\section{Anna M.H. Krebber}

No relationship to disclose

Veerle M.H. Coupé

Research Funding: GlaxoSmithKline (Inst), Novartis (Inst)

\section{Pim Cuijpers}

No relationship to disclose

\section{Remco de Bree}

Consulting or Advisory Role: Norgine

Research Funding: Norgine (Inst)

Annemarie Becker-Commissaris

No relationship to disclose

Egbert F. Smit

No relationship to disclose

\section{Annemieke van Straten}

No relationship to disclose

Guus M. Eeckhout

No relationship to disclose

Aartjan T.F. Beekman

No relationship to disclose

C. René Leemans

No relationship to disclose

Irma M. Verdonck-de Leeuw

Consulting or Advisory Role: Red-kite (Inst)

Research Funding: Achmea Health Care Assurance (Inst), Red-kite (Inst) Patents, Royalties, Other Intellectual Property: Oncokompas 2.0, a selfmanagement portal for cancer patients targeting quality of life (Inst) 